\title{
Low-Complexity Iterative Channel Estimation and Tracking for Time-Varying Multi-Antenna Systems
}

\author{
Yavuz Yapicı \\ Dept. of Electrical and Electronics Eng. \\ Middle East Technical University \\ Ankara,TURKEY
}

\author{
A. Özgür Yılmaz \\ Dept. of Electrical and Electronics Eng. \\ Middle East Technical University \\ Ankara,TURKEY
}

\begin{abstract}
The iterative channel estimation and tracking problem is considered for time-varying frequency-flat fading multiinput multi-output (MIMO) systems. In order to jointly estimate and track the time-varying multi-antenna channel, the pilot symbol assisted modulation (PSAM) technique is generalized to the multi-antenna systems. By distributing the pilot symbols along the transmitted block, i.e., PSAM transmission, the proposed system gains the ability to track the channel variation with the same amount of pilots whereas the system with all the pilots are employed prior to the data block has no tracking capability. The error performance is further improved by iteratively estimating the channel which employs the optimal MMSE filtering and also makes use of the soft probabilities of the coded symbols provided by the decoder. In order to decrease the computational complexity due to the iterative usage of MMSE filtering, we introduce the low-complexity 2-way LMS algorithm based on the forward-backward operation of conventional forward only LMS algorithm. By simulations, 2-way LMS is shown to have a near optimal error performance and highly better channel tracking ability than the conventional LMS algorithm with no significant complexity increase.
\end{abstract}

\section{INTRODUCTION}

$\mathbf{T}$ HE improved services offered by next generation wireless systems require high data rate to support the quality of service demands. To this end, deploying multiple antennas at both the transmitter and the receiver side in order to provide spatial diversity is a powerful choice amongst the others. In order to further improve the performance of these MIMO systems, space-time codes which offer a coding structure using spatial and time dimensions mutually are introduced in [1]. Nevertheless, most of these initial work on MIMO communications have the assumption of full knowledge of the channel state information (CSI). Therefore, it is recently of interest to explore for the efficient algorithms to estimate the multi-channel transmission environment.

There are various works in which MIMO channel is estimated under quasi-static conditions, i.e., the channel remains unchanged during a transmitted symbol block [2]. As the coherence time becomes smaller, the variation over a block could not be ignored any more [3]. Under this time-varying channel scenario, a tracking method should be employed if pilot symbols are used as a preamble at the beginning of

This work was supported in part by the Scientific and Technological Research Council of Turkey (TUBITAK) under grant 104E027, and by STM Defence Technologies Engineering Inc. the transmitted block. The Kalman or RLS (Recursive Least Squares) type well-known tracking algorithms, both of which come with the increased computational complexity, are offered to track the gain and phase variations of the multi-channel fading environment [4]. As an alternative, it is of interest in this paper to distribute the pilot symbols along the transmitted block and use them in channel tracking to get rid of the aforementioned tracking algorithms with high complexity.

In this work, we consider the low-complexity iterative channel estimation (ICE) and tracking algorithms over the coded time-varying flat fading MIMO channel. The proposed algorithms are of data-aided type in the sense that initial channel estimation is computed based on known pilot symbols. The overall estimation mechanism is an iterative procedure such that the soft, or possibly hard, estimates of the decoded symbols are employed in subsequent iterations to further refine the channel estimate. The contribution of this work is twofold. First, the pilot-symbol assisted modulation (PSAM) [5] is proposed as the transmission technique which does not only estimate the multi-channel but also tracks the variations in complex channel taps jointly without any necessity for high complexity tracking algorithms, i.e., Kalman and RLS. The basis of this approach in multi-antenna systems is introduced in [6] and further elaborated by the present authors in [7] for single-antenna systems. As a second contribution, a low-complexity adaptive algorithm called 2-way LMS (Least Mean-Squares) which is a powerful extension of the conventional LMS algorithm is proposed to be used in channel estimation after the initial iteration instead of optimal MMSE (Minimum Mean-Square Estimate) filtering which has excess complexity especially for multi-antenna systems.

\section{SYSTEM MODEL}

\section{A. Channel Model}

We consider a MIMO communication system with $M$ transmitter and $N$ receiver antennas with a time-varying frequencyflat Rayleigh fading channel. The discrete-time complex baseband equivalent channel model under consideration is given at time $k$ by

$$
\mathbf{y}_{k}=\mathbf{H}_{k} \mathbf{s}_{k}+\mathbf{v}_{k}
$$

where $\mathbf{H}_{k}$ is an $N \times M$ random channel matrix composed of 


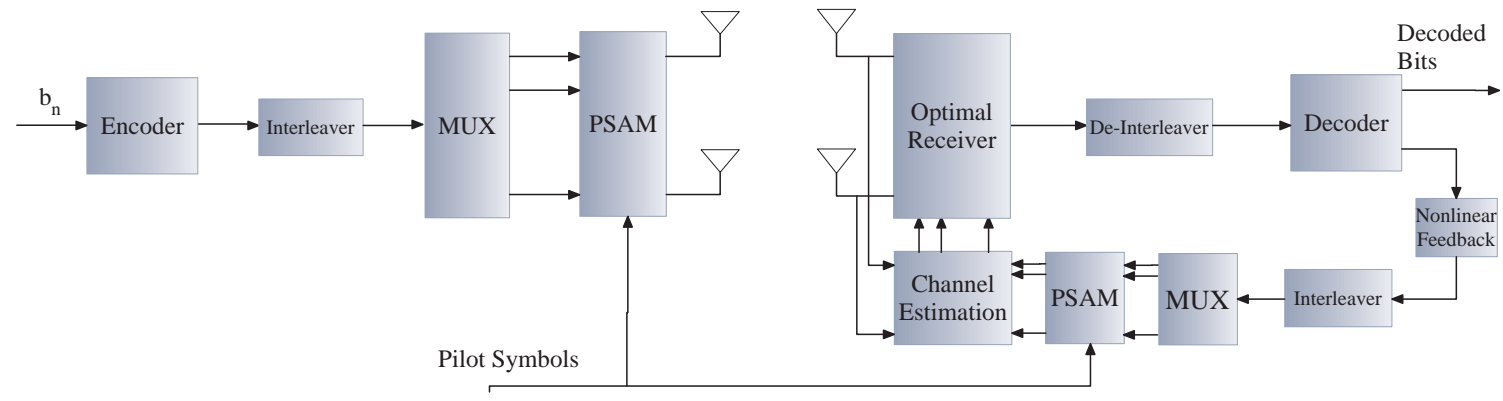

Fig. 1. Transmitter and receiver model

independent complex Gaussian entries $h_{n m, k}$ with zero mean and unit variance, $\mathbf{s}_{k}=\left[\begin{array}{llll}s_{1, k} & s_{2, k} \ldots & s_{M, k}\end{array}\right]^{T}$ is an $M \times 1$ vector of transmitted symbols, $\mathbf{v}_{k}=\left[\begin{array}{llll}v_{1, k} & v_{2, k} \ldots & v_{N, k}\end{array}\right]^{T}$ is an $N \times 1$ noise vector composed of independent and identically distributed complex Gaussian random variables with zero mean and variance $N_{0}$, and $\mathbf{y}_{k}=\left[\begin{array}{lll}y_{1, k} & y_{2, k} \ldots y_{N, k}\end{array}\right]^{T}$ is the corresponding $N \times 1$ complex vector of channel observations. The channel matrix $\mathbf{H}_{k}$ is assumed to be unknown at both the transmitter and the receiver, and independent of the transmitted symbols and noise vector. In addition, the noise variance $N_{0}$ and the maximum Doppler frequency $f_{D}$ is assumed to be known at the receiver.

We may express the channel given by (1) in terms of each received symbol at time $k$ as

$$
y_{n, k}=\sum_{m=1}^{M} h_{n m, k} s_{m, k}+v_{n, k}, \quad n=1,2, \ldots, N
$$

Assuming the unit energy transmitted symbols, i.e., $\mathbb{E}\left\{\left|s_{m, k}\right|^{2}\right\}=1$, the resulting average received symbol signalto-noise ratio (SNR) at each of the receiver antenna is then given by

$$
\gamma_{s}=\frac{M \mathbb{E}\left\{\left|h_{n m, k}\right|^{2}\right\} \mathbb{E}\left\{\left|s_{m, k}\right|^{2}\right\}}{N_{0}}=\frac{M}{N_{0}}
$$

and the corresponding information bit SNR is $\gamma_{b}=\gamma_{s} / R_{T}$ where $R_{T}$ is the overall transmission rate of the system.

\section{B. Transmitter and Receiver Models}

At the transmitter shown in Fig. 1, a random data block $\left\{b_{n}\right\} \in\{-1,+1\}, 1 \leq n \leq L$, is first encoded by a channel code and the produced coded symbols $\left\{c_{n}\right\}, 1 \leq n \leq L / R_{T}$, are then interleaved by the permutation $\Pi$ before going through the spatial multiplexer. At the output of the multiplexer, the coded symbol vectors $\left\{\mathbf{s}_{d, l}\right\}, 1 \leq l \leq L_{d}$ are parsed into the blocks of $P-1$ vectors and a known pilot symbol vector $\left\{\mathbf{s}_{p, l}\right\}$, $1 \leq l \leq L_{p}$, is inserted into the center of each of the block to produce the overall symbol vectors $\left\{\mathbf{s}_{k}\right\}, 1 \leq k \leq L_{T}$, to be transmitted. In this formulation, $P$ is defined as the pilot symbol vector spacing, $L_{d}=L /\left(M R_{T}\right)$ is the total number of coded symbol vector, $L_{p}=L_{d} /(P-1)$ is the total number of pilot symbol vectors, and $L_{T}=L_{d}+L_{p}$ is the total number of transmitted vectors including both the coded and the pilot symbol vectors. This methodology is indeed a generalization of the PSAM (Pilot Symbol Assisted Modulation) technique proposed for the single-antenna systems in [5].

At the receiver, the observation vectors $\left\{\mathbf{r}_{k}\right\}, 1 \leq k \leq L_{T}$, are combined together in order to compute the log-likelihood ratios (LLRs) of the coded symbols to be used by the softinput soft-output decoder. The LLRs of the coded symbols are computed in the optimal sense as done in [8] with a difference, which does not affect the formulation of the LLRs, that is the underlying channel in this work is time-varying and unknown. Therefore, the LLRs computation in the present work employs also the output of the channel estimation unit. The initial channel estimation is performed by using only pilot symbol vectors, whereas this estimate is further refined in the subsequent channel estimation iterations by employing the soft probabilities on the coded symbols provided by the decoder. The feedback mechanism considered in this work is of soft kind whereas the hard estimates could also be used.

\section{PSAM IN ITERATIVE MIMO ChANNEL ESTIMATION}

Using all the pilot symbols at the beginning of the transmitted block, i.e., training sequence, is a good choice for the quasi-static channels in which the fading level remains unchanged during the transmission of a block. However, this method requires additional channel tracking algorithms with high complexity if the channel under consideration has a timevarying nature. In this section, we propose to use PSAM technique in order to jointly estimate and track the MIMO channel. This methodology offers to distribute the same amount of pilot symbols along a transmitted block rather than using them at the beginning of the block, and eventually removes the necessity for computationally complex tracking algorithms.

In the time-varying multi-antenna system under consideration, each subchannel between a transmitter and a receiver antenna pair has a Rayleigh fading with the combined spatial and temporal autocorrelation according to the Jakes's model given as

$$
\mathbb{E}\left\{h_{n m, k} h_{n^{\prime} m^{\prime}, l}\right\}=\delta_{n n^{\prime}} \delta_{m m^{\prime}} J_{o}\left(2 \pi f_{D}(k-l) T_{s}\right)
$$

where $J_{0}()$ is the Bessel function of zeroth order, $T_{s}$ is the symbol duration, and $\delta_{i j}$ is the delta function which is 1 if 
$i=j$ and 0 otherwise.

In order to estimate a complex subchannel value $h_{n m, k}$, one should consider many and possibly all correlated values of this subchannel during a transmission block. As a result, the set $h_{n m, k}, 1 \leq k \leq L_{T}$, should be estimated jointly. Furthermore, since each observation symbol at a single receiver antenna has a partial information on all subchannels terminating at that antenna according to (2), then the set $h_{n m, k}, 1 \leq k \leq L_{T}$, $1 \leq m \leq M$ should be estimated jointly for optimal operation. In order to obtain the sufficient statistics for this estimation, let us modify the expression (2) as

$$
y_{n, k}=\mathbf{s}_{k}^{T} \mathbf{h}_{n, k}+\mathbf{v}_{k} .
$$

where $\mathbf{h}_{n, k}^{T}=\left[h_{n 1, k} h_{n 2, k} \ldots h_{n M, k}\right]$ is the $n$-th row of the channel matrix at time $k$. When the time variation is also considered, we obtain

$$
\mathbf{y}_{n}=\mathbf{S} \mathbf{h}_{n}+\mathbf{v}_{n},
$$

where $\mathbf{y}_{n}=\left[y_{n, 1} y_{n, 2} \ldots y_{n, L_{T}}\right]^{T}$ is the received symbol vector and $\mathbf{v}_{n}=\left[v_{n, 1} v_{n, 2} \ldots v_{n, L_{T}}\right]^{T}$ is the noise vector for $n$-th antenna during the transmission of a block. The random

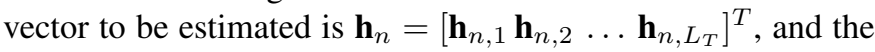
transmitted symbol matrix $\mathbf{S}$ is defined as

$$
\mathbf{S}=\left[\begin{array}{cccc}
\mathbf{s}_{1}^{T} & \mathbf{0}^{T} & \ldots & \mathbf{0}^{T} \\
\mathbf{0}^{T} & \mathbf{s}_{2}^{T} & \ldots & \mathbf{0}^{T} \\
\vdots & \vdots & \vdots & \vdots \\
\mathbf{0}^{T} & \mathbf{0}^{T} & \ldots & \mathbf{s}_{L_{T}}^{T}
\end{array}\right]
$$

The linear MMSE estimator of $\mathbf{h}_{n}$ for the known transmitted symbol matrix $\mathbf{S}$ is then given by [9]

$$
\hat{\mathbf{h}}_{n, M M S E}=\mathbf{R} \mathbf{S}^{H}\left(\mathbf{S R S}^{H}+N_{0} \mathbf{I}\right)^{-1} \mathbf{y}_{n},
$$

where $\mathbf{I}$ is the identity matrix, the superscript ()$^{H}$ denotes the Hermitian operation, and the channel correlation matrix $\mathbf{R}$ is defined as $\mathbf{R}=\mathbb{E}\left\{\mathbf{h}_{n} \mathbf{h}_{n}^{H}\right\}$ and is computed using (4).

Note that, initially the receiver only knows the pilot symbol vectors $\left\{\mathbf{s}_{p, l}\right\}, 1 \leq l \leq L_{p}$. Therefore, we construct an alternative form for transmitted symbol matrix by replacing the unknown coded symbol vectors with the nearest pilot symbol vector, which is a similar idea for single-antenna systems handled in [6]. The resulting alternative form is then given by

$$
\mathbf{S}_{p}=\left[\begin{array}{cccc}
\mathbf{S}_{p 1} & \mathbf{0}^{T} & \ldots & \mathbf{0}^{T} \\
\mathbf{0}^{T} & \mathbf{S}_{p 2} & \ldots & \mathbf{0}^{T} \\
\vdots & \vdots & \vdots & \vdots \\
\mathbf{0}^{T} & \mathbf{0}^{T} & \ldots & \mathbf{S}_{p L_{p}}
\end{array}\right]
$$

where $\mathbf{S}_{p l}, 1 \leq l \leq L_{p}$, is a $P \times P$ square matrix given as $\mathbf{S}_{p l}=\mathbf{s}_{p, l}^{T}$ I. Note that, we should also modify the observation vector $\mathbf{y}_{n}$ in (7) so that it becomes compatible with the alternative form for the transmitted symbol matrix $\mathbf{S}_{p}$. To this end, we define the modified observation vector $\mathbf{y}_{p, n}$ as

$$
\mathbf{y}_{p, n}=\left[\begin{array}{c}
y_{n, l_{1}} \mathbf{u}^{T} \\
y_{n, l_{2}} \mathbf{u}^{T} \\
\vdots \\
y_{n, l_{L_{p}}} \mathbf{u}^{T}
\end{array}\right],
$$

where the set $\left\{l_{1}, l_{2}, \ldots, l_{L_{p}}\right\}$ contains the time indices of the transmitted pilot symbol vectors, and $\mathbf{u}$ is a $P \times 1$ column vector with all entries 1 . The resulting initial MMSE estimate is then given as

$$
\hat{\mathbf{h}}_{n, M M S E}=\mathbf{R} \mathbf{S}_{p}^{H}\left(\mathbf{S}_{p} \mathbf{R} \mathbf{S}_{p}^{H}+N_{0} \mathbf{I}\right)^{-1} \mathbf{y}_{p, n} .
$$

Subsequently, LLRs of the coded symbols are computed based on initial channel estimate, deinterleaved and fed to the decoder which computes the extrinsic information, $\lambda_{n}$, of the coded symbols. The expected value of these soft probabilities, i.e., $\tanh \left(\lambda_{n}\right)$, are then interleaved, multiplexed and used as if they are the actual transmitted coded symbols. In the subsequent channel estimation iterations, the resulting estimates of the coded symbols are used together with the pilot symbols to obtain a refined estimate of the channel according to (7), in an iterative fashion.

\section{LOW-COMPLEXITY ICE FOR MIMO TRANSMISSION}

The MMSE channel estimation in multi-antenna systems has a high computational complexity which makes this optimal choice impractical. In the literature, conventional forward only LMS, which we called l-way LMS to differentiate with the modified version introduced in this section, is offered for ICE algorithms in single-antenna systems to reduce the computational complexity. However, the tracking capability of this conventional LMS is shown to be poor for singleantenna systems in [7], and also for multi-antenna systems in Section V, under fast time-varying fading. In this section, we therefore propose a modified LMS algorithm called 2-way $L M S$ which has an improved, indeed near optimal, tracking capability for fast time-varying multi-antenna systems with no significant complexity increase with respect to the conventional one.

\section{A. 1-way LMS Algorithm}

In this section, assuming that the initial channel estimate was computed according to (8) and the decoder has produced the soft probabilities on coded symbols, we derive the 1-way LMS algorithm in order to estimate the MIMO channel in the subsequent channel estimation iterations. To this end, the error term $e_{n, k}^{F}$ in the forward direction for the received symbol $y_{n, k}$ is defined as

$$
e_{n, k}^{F}=y_{n, k}-\left(\hat{\mathbf{s}}_{k}^{(i-1)}\right)^{T} \hat{\mathbf{h}}_{n, k}^{(i)}
$$

where $\hat{\mathbf{s}}_{k}^{(i-1)}$ denotes the soft estimate of the coded symbol vector $\hat{\mathbf{s}}_{k}$ at the $(i-1)$-th iteration, and $\hat{\mathbf{h}}_{n, k}^{(i)}$ denotes the estimate for the $n$-th row of the channel matrix in the forward direction at time $k$. The corresponding update equation for the $n$-th row of the channel matrix is therefore given as 


$$
\hat{\mathbf{h}}_{n, k+1}^{(i)}=\hat{\mathbf{h}}_{n, k}^{(i)}+\beta e_{n, k}^{F}\left(\hat{\mathbf{s}}_{k}^{(i-1)}\right)^{T} .
$$

Further elaborating this expression such that it involves all of the rows gives us

$$
\hat{\mathbf{H}}_{k+1}^{i}=\hat{\mathbf{H}}_{k}^{i}+\beta \mathbf{e}_{k}^{F}\left(\hat{\mathbf{s}}_{k}^{(i-1)}\right)^{T}
$$

where $\hat{\mathbf{H}}_{k}^{i}$ stands for the estimate of the channel matrix at the $i$-th iteration and time index $k$, the error vector in the forward direction is defined as $\mathbf{e}_{k}^{F}=\left[e_{1, k}^{F} e_{2, k}^{F} \ldots e_{N, k}^{F}\right]^{T}$, and $\beta$ denotes the step size of the adaptive algorithm. Note that, LMS update algorithm in this fashion employs the the estimate of the coded symbols at the $(i-1)$-th estimation iteration to compute the channel estimate at the $i$-th estimation iteration.

\section{B. 2-way LMS Algorithm}

Now, we introduce the 2-way LMS algorithm for the multiantenna systems which operates the forward only LMS both in the forward and the backward direction along the transmitted block. Again, assuming that the initial channel estimate is obtained and the soft information on coded symbols are accordingly produced by the decoder, the 2-way LMS algorithm computes the forward channel estimates $\hat{\mathbf{F}}_{k}^{i}$ using (11). The backward channel estimate $\hat{\mathbf{G}}_{k}^{i}$ at the $i$-th estimation iteration is computed by employing the conventional LMS algorithm in the reverse direction along the transmitted block as follows

$$
\hat{\mathbf{G}}_{k-1}^{i}=\hat{\mathbf{G}}_{k}^{i}+\beta \mathbf{e}_{k}^{B}\left(\hat{\mathbf{s}}_{k}^{(i-1)}\right)^{T} .
$$

The corresponding error vector in the backward direction is defined as $\mathbf{e}_{k}^{B}=\left[e_{1, k}^{B} e_{2, k}^{B} \ldots e_{N, k}^{B}\right]$ with the entries

$$
e_{n, k}^{B}=y_{n, k}-\left(\hat{\mathbf{s}}_{k}^{(i-1)}\right)^{T} \hat{\mathbf{g}}_{n, k}^{(i)}
$$

where $\hat{\mathbf{g}}_{n, k}^{(i)}$ is the $n$-th row of $\hat{\mathbf{G}}_{k}^{i}$. The overall MIMO channel estimate is defined to be a linear function of the estimates in the forward and backward directions. For simplicity, we choose the arithmetic average as the linear operation which results in the following channel estimate for the 2-way LMS algorithm at time $k$

$$
\hat{\mathbf{H}}_{k}^{i}=\left(\hat{\mathbf{F}}_{k}^{i}+\hat{\mathbf{G}}_{k}^{i}\right) / 2 .
$$

\section{Numerical RESUlts}

We consider a fast time-varying frequency-flat multiantenna system employing 2 transmitter and 4 receiver antennas with the maximum Doppler frequency $f_{D}=100 \mathrm{~Hz}$, the symbol time $T_{s}=0.1 \mathrm{~ms}$, the pilot symbol vector spacing $P=7$, and the data length $L=98$. The resulting total number of transmitted symbol vector is therefore $L_{d}=100$ including trellis termination bits, and the normalized Doppler frequency along a whole block is $f_{D} L_{T}=1.17$ which corresponds to a significant time-variation. Pilot symbols are assumed to be zero-mean and unit variance independent Gaussian random variables. The data block is encoded by a convolutional code

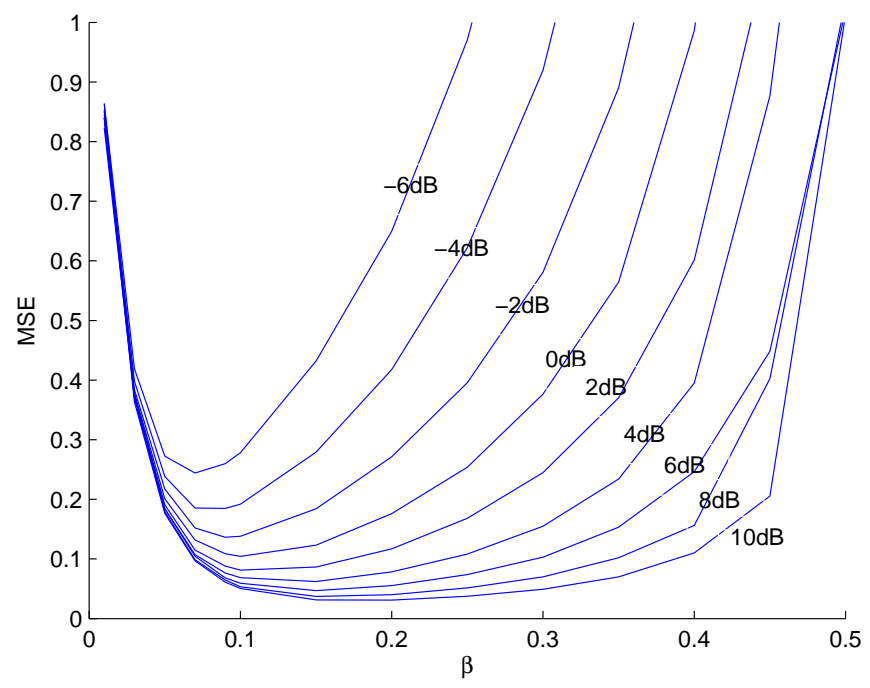

Fig. 2. Average MSE for 2-way LMS for varying step-size, i.e., $\beta \epsilon\{0, \ldots, 0.5\}$, and received symbol SNR, $\gamma_{s} \epsilon\{-6 \mathrm{~dB}, \ldots, 10 \mathrm{~dB}\}$. Each bowl-type curve is for a specific value of $\gamma_{s}$.

with generator $(1,5 / 7)_{8}$, and the channel interleaver is the random interleaver. The number of channel estimation iteration is 5 which provides a satisfactory convergence.

In order to assess the channel tracking performances, we first evaluate the average mean-square error (MSE), i.e., $\mathbb{E}\left\{\left\|\mathbf{H}_{k}-\hat{\mathbf{H}}_{k}\right\|^{2}\right\}$, under full knowledge of transmitted symbols (genie-aided) condition. For this purpose, the optimal tracking performance of both the 1-way LMS and 2-way LMS algorithms are obtained for varying step-size $(\beta)$ and received symbol SNR $\left(\gamma_{s}\right)$ values, and shown in Fig. 2 for 2-way LMS, as an example. The optimal $\beta$ values are chosen accordingly and then used in average MSE computation. The result is shown in Fig. 3. It is observed that the performance of LMSbased algorithms are highly dependent on $\beta$ choice, and that tracking capability of proposed 2-way LMS algorithm is near that of the optimal MMSE whereas 1-way LMS achieves a poor tracking performance especially at the low SNR regime.

As a second comparison, the effect of coding is considered on the channel estimation performance in an iterative fashion under a more realistic situation where only the pilot symbols are known a-priori, and LMS-based algorithms operate with the optimal $\beta$ values chosen on trial and error basis for each SNR level. We make a number of observations from the corresponding simulation results shown in Fig. 4. First, the assumption made in [10] that the received noisy codeword in multi-antenna systems which is also corrupted by fading may be decoded using the channel estimate at the beginning of the block does not work under this fast time-varying scenario. As shown in the figure, even for the exact value of the channel matrix at the beginning of the block, i.e., $\mathbf{H}_{0}$, fails to decode the whole transmitted block. Secondly, the proposed PSAM technique for the multi-antenna systems achieves a good tracking ability with its low-complexity structure, even for the 


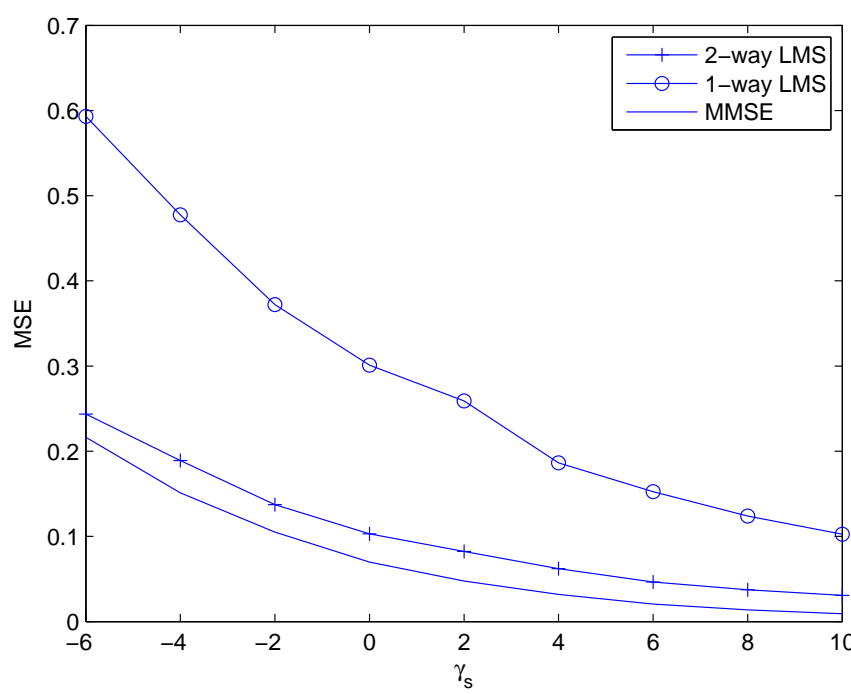

Fig. 3. Average MSE for MMSE, 1-way LMS and 2-way LMS algorithms under full knowledge of transmitted symbols (genie-aided). LMS-based algorithms use the optimal $\beta$ 's.

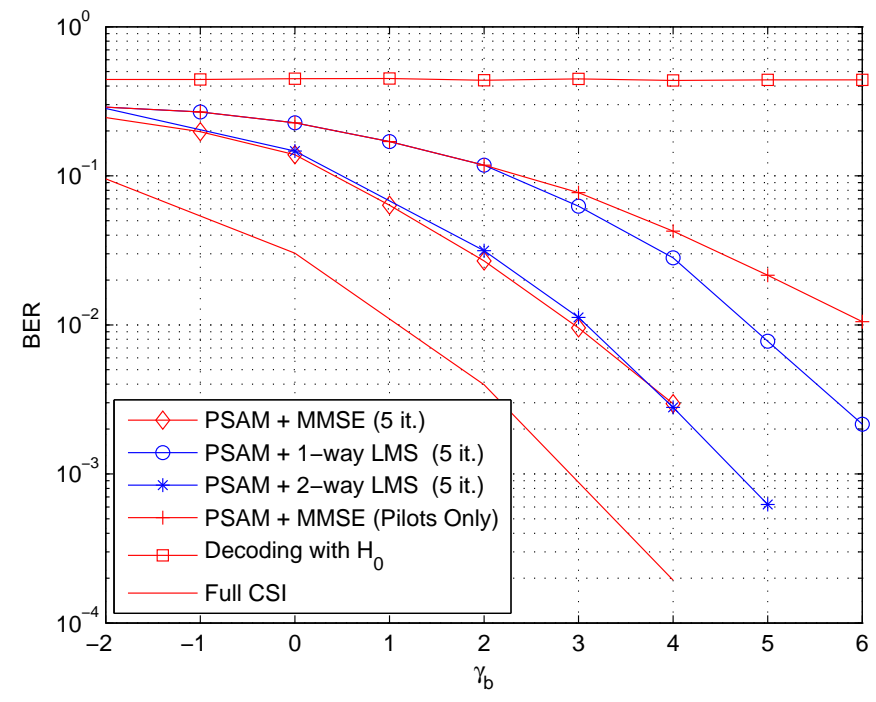

Fig. 4. BER for known channel, PSAM with MMSE which uses pilots only, and PSAM with MMSE, 1-way LMS, and 2-way LMS algorithms together with the soft probabilities on the coded symbols. Total number of iterations for channel estimation is 5 . Decoding performance with $\mathbf{H}_{0}$ is also provided.

initial channel estimation which employs the pilot symbols only. Thirdly, the performance of PSAM is further improved by iteratively estimating the channel and incorporating the soft estimates of the coded symbols via 2-way LMS algorithm which achieves almost the same error performance with the optimal MMSE approach. The error performance of 2-way LMS and MMSE pair is off the known case by $1.5 \mathrm{~dB}$ whereas this penalty for 1-way LMS is approximately $4 \mathrm{~dB}$ at $\mathrm{BER}=10^{-3}$.

\section{CONCLUSION}

In this work, the joint iterative channel estimation and tracking problem is considered for time-varying frequency-flat fading multi-antenna transmission. We generalize the PSAM technique to the multi-antenna systems and show that it has the capability of tracking the variation in the channel fading level with the same amount of pilot symbols as compared to the training structures where all the pilot symbols are in the form of a preamble sequence. It is also shown that the generalized PSAM achieves the satisfactory error performance without any necessity for the special tracking algorithms such as RLS and Kalman filtering both of which are computationally expensive. We further improve the error performance of the PSAM transmission by iteratively estimating the channel and incorporating the soft estimates of the coded symbols. In order to decrease the resulting complexity of the iterative channel estimation structure, we propose to use the low-complexity 2-way LMS algorithm after initial channel estimation instead of optimal high-complexity MMSE filtering. The 2-way LMS algorithm, which operates in both the forward and the backward directions along a transmitted block, is shown to achieve a similar error performance to that of the optimal MMSE filtering, and to have much better error performance than the conventional 1-way LMS algorithm with no significant complexity increase.

\section{REFERENCES}

[1] V. Tarokh, N. Seshadri, and A. Calderbank, "Space-time codes for high data rate wireless communication: Performance criterion and code construction," IEEE Trans. Inf. Theory, vol. 44, no. 2, pp. 744-765, Mar 1998.

[2] T. L. Marzetta, "Blast training: Estimating channel characteristics for high capacity space-time wireless," in Proc. of 37th Annual Allerton Conference, 1999, pp. 958-966.

[3] Q. Sun, D. Cox, A. Lozano, and H. Huang, "Training-based channel estimation for continuous flat fading BLAST," in Proc. of ICC, vol. 1, Apr-May 2002, pp. 325-329.

[4] C. Komninakis, C. Fragouli, A. Sayed, and R. Wesel, "Multi-input multi-output fading channel tracking and equalization using Kalman estimation," IEEE Trans. Signal Process., vol. 50, no. 5, pp. 1065-1076, May 2002.

[5] J. K. Cavers, "An analysis of pilot symbol assisted modulation for rayleigh fading channels," IEEE Trans. Veh. Technol., vol. 40, pp. 686693, Nov. 1991.

[6] M. C. Valenti and B. D. Woerner, "Iterative channel estimation and decoding of pilot symbol assisted turbo codes over flat-fading channels," IEEE J. Sel. Areas Commun., vol. 19, pp. 1697-1705, Sep. 2001.

[7] Y. Yapıcı and A. Ö. Yılmaz, "Joint channel estimation and decoding with low-complexity iterative structures in time-varying fading channels," in Proc. of IEEE PIMRC'09, Tokyo, Japan, Sep. 13-16, 2009.

[8] A. Stefanov and T. Duman, "Turbo-coded modulation for systems with transmit and receive antenna diversity over block fading channels: System model, decoding approaches, and practical considerations," IEEE J. Sel. Areas Commun., vol. 19, no. 5, pp. 958-968, May 2001.

[9] S. Haykin, Adaptive Filter Theory (4th Edition). Prentice Hall, September 2001.

[10] E. Eitel and J. Speidel, "Enhanced decision-directed channel estimation of time-varying flat MIMO channels," in Proc. of PIMRC'07, Sep 2007, pp. $1-5$. 